\title{
Evaluation of Clinical Outcomes after LASIK for the Correction of Myopia Using the Advanced Customvue Excimer Laser Platform
}

\author{
Hung Ming Lee \\ Lee Hung Ming Eye Centre, Gleneagles Hospital, Singapore \\ Email: lasik28@yahoo.com.sg, contact@leehmeyecentre.com.sg
}

How to cite this paper: Lee, H.M. (2018) Evaluation of Clinical Outcomes after LASIK for the Correction of Myopia Using the Advanced Customvue Excimer Laser Platform. Open Journal of Ophthalmology, 8, 150-160.

https://doi.org/10.4236/oioph.2018.83019

Received: May 18, 2018

Accepted: July 30, 2018

Published: August 3, 2018

Copyright $\odot 2018$ by author and Scientific Research Publishing Inc. This work is licensed under the Creative Commons Attribution International License (CC BY 4.0).

http://creativecommons.org/licenses/by/4.0/

\section{(c) (†) Open Access}

\begin{abstract}
Purpose: To evaluate early visual, refractive and aberrometric outcomes after wave front-guided LASIK for the correction of low to moderate myopia and myopic astigmat is musing the excimer laser platform Advanced Custom Vue. Methods: A prospective, non-comparative study in 100 myopic eyes of 50 patients evaluating LASIK results over a period of 3 months. Main outcome variables included visual acuity, refraction, total higher-order aberrations (HOAs), spherical aberration (SA) and coma-like aberration. The surgery was performed using the VISX STAR S4 (Johnson and Johnson Vision) excimer laser and a wave front-guided ablation designed according to the iDesign aberrometer data (Johnson and Johnson Vision). Results: Mean preoperative sphere decreased from $-4.07 \pm 1.78 \mathrm{D}$ to $0.32 \pm 0.30 \mathrm{D}$ at 3 months after surgery. Mean preoperative cylinder was reduced from $-1.09 \pm 0.88 \mathrm{D}$ to $-0.26 \pm$ $0.28 \mathrm{D}$ after the complete follow-up. Postoperative uncorrected distance visual acuity (UDVA) was $0.00 \log$ MAR (20/20) or better in $100 \%$ of eyes, and $65.3 \%$ of eyes reached an UDVA of $-0.20 \operatorname{logMAR}(20 / 12.5)$ or better $(\mathrm{p}<0.05)$. For a 6-mm pupil, the root mean square (RMS) for total ocular HOAs increased with surgery by $0.03 \mu \mathrm{m}(\mathrm{p}=0.050)$, whereas the coma-like RMS increased by $0.05 \mu \mathrm{m}(\mathrm{p}<0.001)$. The change in spherical aberration was $+0.08 \mu \mathrm{m}(\mathrm{p}<$ 0.001). Conclusion: Wavefront-guided LASIK using the new generation excimer laser platform Advanced Custom Vue is safe and effective for treating myopia and myopic astigmatism, minimizing the postoperative level of HOAs.
\end{abstract}

\section{Keywords}

Excimer Laser, LASIK, Wavefront-Guided, Myopia, Higher-Order Aberrations 


\section{Introduction}

Although it has been demonstrated that Laser in situ keratomileusis (LASIK) procedure is effective and predictable for correcting myopic refractive errors, the induction of higher order aberrations (HOAs) due to changes of the corneal shape is a major concern for practitioners [1]. The oblate cornea, as a result of the myopic laser photoablation, may potentially induce a significant reduction in visual quality and subjective night vision symptoms [2] [3]. In order to improve the postoperative outcomes different algorithms have been developed, and aspheric profiles have been fully incorporated to the different laser platforms to minimize those undesirable optical side effects. The Customvue platform using the iDesign high resolution aberrometer (Advanced CustomVue, Johnson and Johnson Vision) incorporates a wavefront-guided (WF-guided) profile based on the preoperative aberrometry data and specifically on the values of the root mean square (RMS) [4]. This prospective study aims to evaluate the visual outcomes and changes in HOAs after LASIK using the Advanced CustomVue excimer laser platform.

\section{Patients and Methods}

\subsection{Patients}

A prospective consecutive case series was performed at Gleneagles Hospital, Singapore, to evaluate the early visual and aberrometric outcomes after WF-guided myopic LASIK treatments.

All patients underwent a complete preoperative ophthalmological examination including ocular and medical history, measurement of uncorrected distance visual acuity (UDVA), corrected distance visual acuity (CDVA), manifest and cycloplegic refraction, slit-lamp examination, computerized Orbscan to pography (Bausch \& Lomb), pachymetry, applanation tonometry and fundoscopy. Wavefront-aberration measurements (iDesign aberrometer; Johnson and Johnson Vision) were performed to calculate RMS-HOAs, spherical aberration (SA), and primary coma-like aberration for a pupil diameter of $6 \mathrm{~mm}$. If applicable, patients were asked to discontinue the use of soft or rigid gas permeable contact lenses prior to the preoperative examination for at least one or 3 weeks, respectively.

Exclusion criteria for corneal refractive procedure included a myopic spherical equivalent over -10 D, monocular CDVA of less than 20/20, unstable refraction during the last 12 months, inability to return for the scheduled follow-up examinations, diagnosis of dry eye, any corneal opacity, significant pathology of the anterior segment, significant residual, recurrent or active ocular disease, previous intraocular or corneal surgery, history of herpetic keratitis, diagnosis of immunodeficiency, systemic connective tissue diseases or atopic syndrome, insulin-dependent diabetes mellitus, subjects taking systemic medications likely to affect wound healing or vision, unstable or irregular topography readings, specifically corneal ectatic diseases, intraocular pressure over $23 \mathrm{mmHg}$ by Gold- 
mann applanation tonometry, history or suspect of glaucoma, media opacities, iris coloboma or any other irregularity of the pupil margin, and pregnancy or breastfeeding. There was no restriction on patients with large mesopic pupil diameter measured with an infrared pupillometer.

\subsection{Surgical Procedure}

All eyes were treated with a WF-guided ablation profile (Advanced CustomVue) calculated according to the aberrometric measurements obtained with the high-resolution Hartmann-Shack aberrometer iDesign (Johnson and Johnson Vision). This aberrometer is an optimized wavefront sensor based on the WaveS can system. It has been developed with a higher quantity and density of lenslets to allow the analysis of 1257 points for a $7.0 \mathrm{~mm}$-pupil and a higher dynamic range $(-16 \mathrm{D}$ to $+12 \mathrm{D}$ of sphere, 0 to $8 \mathrm{D}$ of cylinder and up to $8 \mu \mathrm{m}$ of $\mathrm{HOA}$ RMS) [5].

The laser ablation was performed using the VISX STAR S4 excimer system (Johnson and Johnson Vision) according to the wavefront aberration data measured preoperatively. Treatments were programmed to ensure complete coverage of the mesopic pupil. The refractive target was emmetropia in all cases. All aberrometric measurements were performed under physiologic conditions in dim illumination by experienced operators and without pupil dilation.

All surgeries were performed by the same surgeon (Dr Lee Hung Ming) under topical anesthesia. Preoperatively, the eyes were prepared by cleansing the periocular zone and instillation of two drops of a topical anesthetic. Corneal flaps were created using the femtosecond laser system (IntraLase, Johnson and Johnson Vision). In all eyes, the VISX STAR S-4 IR laser with a wavefront guided ablation was used considering a torsional registration that was previously performed and applied if necessary. Standard topical postoperative treatment was administered to all patients consisting of a combination of Pred Forte and Cravit 5 times a day for one week, tapering the frequency during the two following weeks. Also, patients were instructed to use an artificial tear solution at least every two hours the day after the surgery and at least four times a day during one month.

\subsection{Postoperative Examinations}

Follow-up examinations were performed one and 3 months after surgery and included UDVA, CDVA, manifest refraction, and aberrometric outcomes for a 6-mm pupil diameter.

\subsection{Statistical Analysis}

Data analysis was performed using the software SPSS for Windows version 19.0 (IBM, Armonk, NY, USA). Normality of data samples was evaluated by means of the Kolmogorov-Smirnov test. When parametric analysis was possible, the Student $t$ test for paired data was used for comparisons between the preoperative and postoperative data, whereas the Wilcoxon rank sum test was applied to as- 
sess the significance of such differences when parametric analysis was not possible. In addition, the Chi-square test was used for comparing percentages between consecutive visits. For all statistical tests, a p-value of less than 0.05 was considered as statistically significant.

\section{Results}

A total of 100 eyes of 50 patients ( 25 females and 25 males) were included in the study. Mean age was $26.22 \pm 7.85$ years.

\subsection{Visual and Refractive Outcomes}

Pre- and postoperative visual acuity and refraction outcomes are summarized in Table 1. Mean logMAR UDVA improved from $1.05 \pm 0.48$ (SD) preoperatively to $-0.04 \pm 0.06$ (SD) at one month and $-0.06 \pm 0.06$ (SD) at 3 months postoperative. P-values associated to the changes between the follow-up periods are summarized in Table 2. Regarding the longitudinal changes in UDVA, statistically significant differences were found between all 3 postoperative visits $(p \leq 0.001)$.

The mean preoperative SE (spherical equivalent) decreased from $-4.63 \mathrm{D} \pm$ 1.79 (SD) to $0.33 \mathrm{D} \pm 0.31(\mathrm{SD})$ and $0.20 \mathrm{D} \pm 0.31$ (SD) one and 3 months after surgery $(\mathrm{p}<0.001)$, respectively. Statistically significant differences were also found in SE between one and 3 months postoperative $(\mathrm{p}=0.002)$. The mean preoperative sphere decreased from $-4.07 \mathrm{D} \pm 1.78$ (SD) to $0.44 \mathrm{D} \pm 0.32$ (SD) and $0.32 \mathrm{D} \pm 0.30(\mathrm{SD})$ one and 3 months postoperatively, respectively ( $\mathrm{p}<$ 0.001). Small in magnitude but statistically significant differences were found in sphere between one and 3 months postoperative $(\mathrm{p}=0.012)$. The mean preoperative cylinder decreased from $-1.09 \mathrm{D} \pm 0.88$ (SD) to $-0.21 \mathrm{D} \pm 0.27$ (SD) and $-0.26 \mathrm{D} \pm 0.28(\mathrm{SD})$ one and 3 months after surgery, respectively $(\mathrm{p}<0.001)$. No statistically significant differences in cylinder were found between the one and 3 -month postoperative examinations $(\mathrm{p}=0.051)$. Regarding predictability, all

Table 1. Preoperative and postoperative data.

\begin{tabular}{cccc}
\hline & $\begin{array}{c}\text { Mean (SD) } \\
\text { Median (Range) } \\
\text { Preoperative }\end{array}$ & $\begin{array}{c}\text { Mean (SD) } \\
\text { Median (Range) } \\
\text { 1-month follow-up }\end{array}$ & $\begin{array}{c}\text { Mean (SD) } \\
\text { Median (Range) } \\
\text { 3-months follow-up }\end{array}$ \\
\hline LogMAR UDVA & $1.05(0.48)$ & $-0.04(0.06)$ & $-0.06(0.06)$ \\
Sphere (D) & $0.90(0.00$ to 2.00$)$ & $0.00(-0.10$ to 0.10$)$ & $-0.10(-0.10$ to 0.10$)$ \\
Cylinder (D) & $-4.07(1.78)$ & $0.44(0.32)$ & $0.32(0.30)$ \\
& $-4.00(-9.25$ to -0.25$)$ & $0.50(-0.50$ to 1.25$)$ & $0.25(-0.50$ to 1.25$)$ \\
Spherical equivalent & $-1.09(0.88)$ & $-0.21(0.27)$ & $-0.26(0.28)$ \\
(D) & $-4.00(-4.75$ to 0.00$)$ & $-0.13(-1.25$ to 0.00$)$ & $-0.25(-1.25$ to 0.00$)$ \\
LogMAR CDVA & -10.00 to -1.13$)$ & $0.25(-0.50$ to 1.12$)$ & $0.25(-0.75$ to 0.88$)$ \\
& $0.00(0.00$ to 0.00$)$ & $-0.10(-0.10$ to 0.00$)$ & $-0.10(-0.10$ to 0.00$)$ \\
\hline
\end{tabular}

Abbreviations: UDVA, uncorrected distance visual acuity; CDVA, corrected distance visual acuity; SD, standard deviation; D, diopter. 
Table 2. P-values associated to specific longitudinal changes in the analyzed sample.

\begin{tabular}{|c|c|}
\hline & p-value \\
\hline \multicolumn{2}{|l|}{ LogMAR UDVA } \\
\hline Preop-1 month & $<0.001$ \\
\hline 1 month-3 months & 0.001 \\
\hline Preop-3 months & $<0.001$ \\
\hline \multicolumn{2}{|l|}{ Sphere (D) } \\
\hline Preop-1 month & $<0.001$ \\
\hline 1 month-3 months & 0.012 \\
\hline Preop-3 months & $<0.001$ \\
\hline \multicolumn{2}{|l|}{ Cylinder (D) } \\
\hline Preop-1 month & $<0.001$ \\
\hline 1 month-3 months & 0.051 \\
\hline Preop-3 months & $<0.001$ \\
\hline \multicolumn{2}{|l|}{ Spherical equivalent (D) } \\
\hline Preop-1 month & $<0.001$ \\
\hline 1 month-3 months & 0.002 \\
\hline Preop-3 months & $<0.001$ \\
\hline \multicolumn{2}{|l|}{ LogMAR CDVA } \\
\hline Preop-1 month & $<0.001$ \\
\hline 1 month-3 months & 0.004 \\
\hline Preop-3 months & $<0.001$ \\
\hline
\end{tabular}

Abbreviations: UDVA, uncorrected distance visual acuity; CDVA, corrected distance visual acuity; SD, standard deviation.

eyes had a SE within $\pm 1 \mathrm{D}$ along the complete follow-up and $92 \%$ of eyes were within $\pm 0.50 \mathrm{D}$ at the end of the follow-up (Figure 1). Figure 2 shows a scattergram demonstrating the high correlation factor $\left(\mathrm{r}^{2}\right)$ between the intended and the attempted refractive outcome.

Postoperative UDVA was $0.00 \log$ MAR (20/20 Snellen scale) or better in $100 \%$ of eyes and $65.3 \%$ of eyes reached a 3-month postoperative UDVA of -0.20 logMAR (20/12.5 Snellenscale) or better (Figure 3). As expected, the improvement in UDVA after surgery was statistically significant $(\mathrm{p}<0.05)$, while the change in CDVA was not statistically significant $(\mathrm{p}>0.05)$ (Figure 4$)$. Regarding safety, no eyes lost lines of CDVA and $73 \%$ of eyes gained one line of CDVA at the end of the follow-up (Figure 5).

\subsection{Aberrometric Outcomes}

Preoperatively, total ocular HOA RMS (6-mm pupil) was $0.20 \mu \mathrm{m} \pm 0.06$ (SD), spherical aberration was $-0.05 \mu \mathrm{m} \pm 0.11$ (SD) and coma-like aberration was $0.10 \mu \mathrm{m} \pm 0.06(\mathrm{SD})$. After 3 months, the induction of total ocular HOA was 


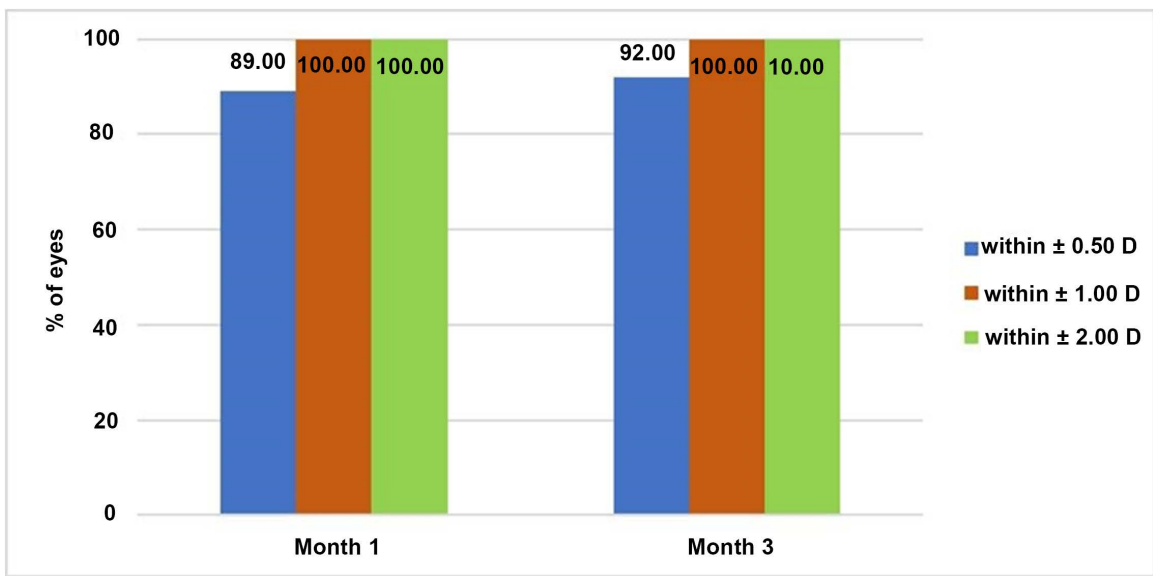

Figure 1. Predictability outcomes in the analyzed sample.

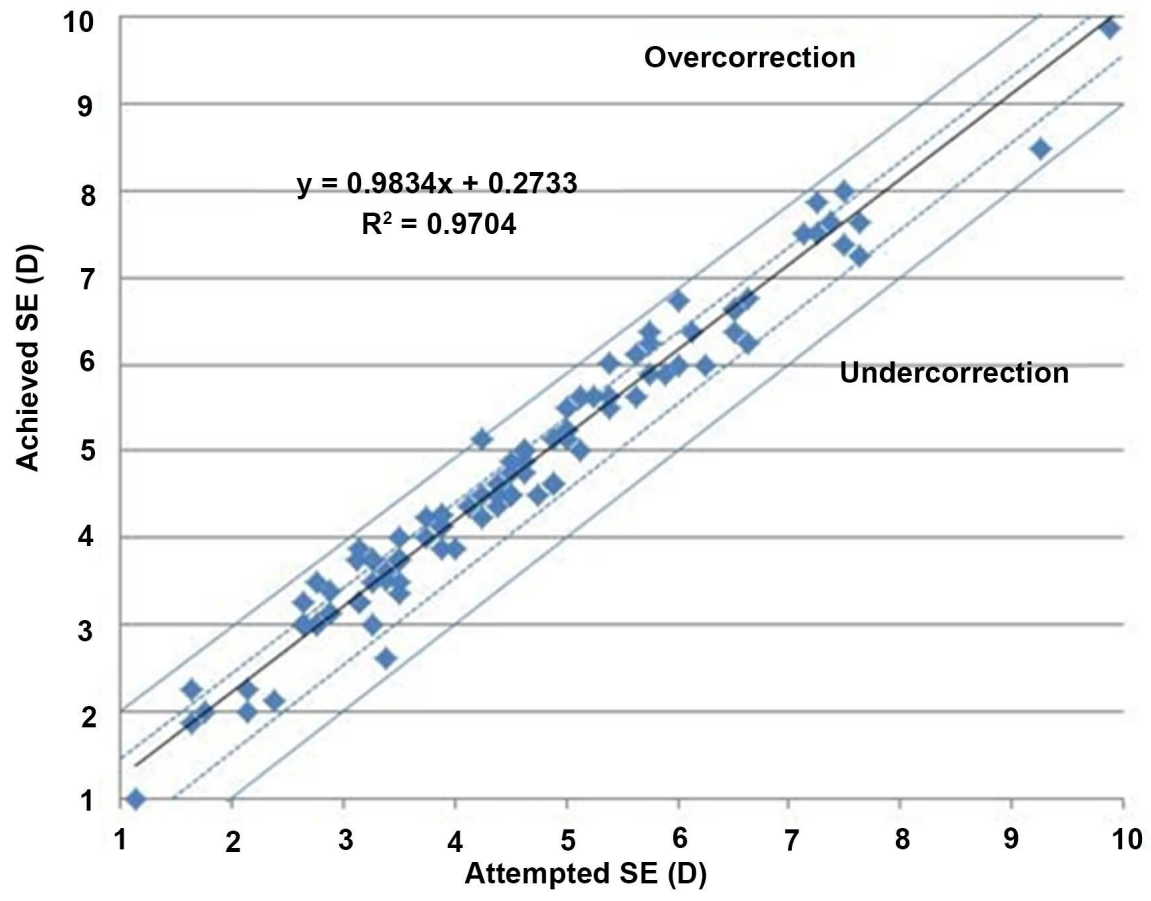

Figure 2. Scattergram showing the relationship between attempted and achieved spherical equivalent correction.

statistically not significant $(\mathrm{p}=0.050)$. Total ocular HOA RMS increased to 0.23 $\mu \mathrm{m} \pm 0.10$ (SD), spherical aberration changed to $0.03 \mu \mathrm{m} \pm 0.08$ (SD) nd coma-like aberration RMS increased to $0.15 \mu \mathrm{m} \pm 0.11$ (SD) (p < 0.01) (Table 3).

\section{Discussion}

The ViSX Star laser system has widely demonstrated its safety and efficacy in the correction of refractive errors using different surgical techniques, such as photorefractive keratectomy (PRK) [6] [7] and laser in situ keratomileusis (LASIK) [8] [9]. However, it is well-known that conventional procedures induce significant amounts of higher-order aberrations (HOA), especially spherical and 


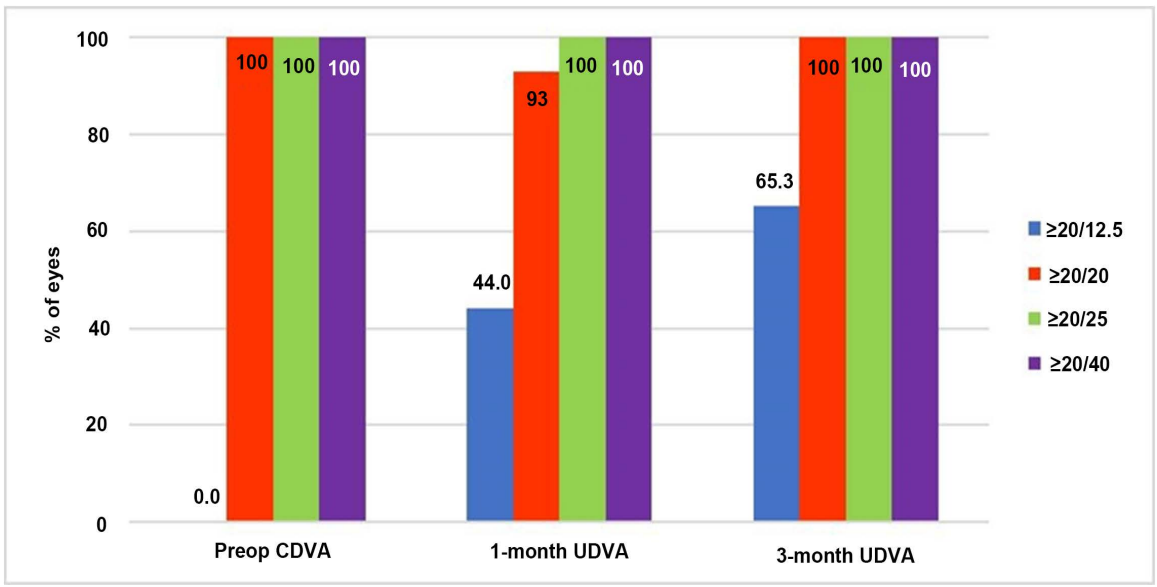

Figure 3. Distribution of preoperative CDVA and postoperative UDVA in the analyzed sample.

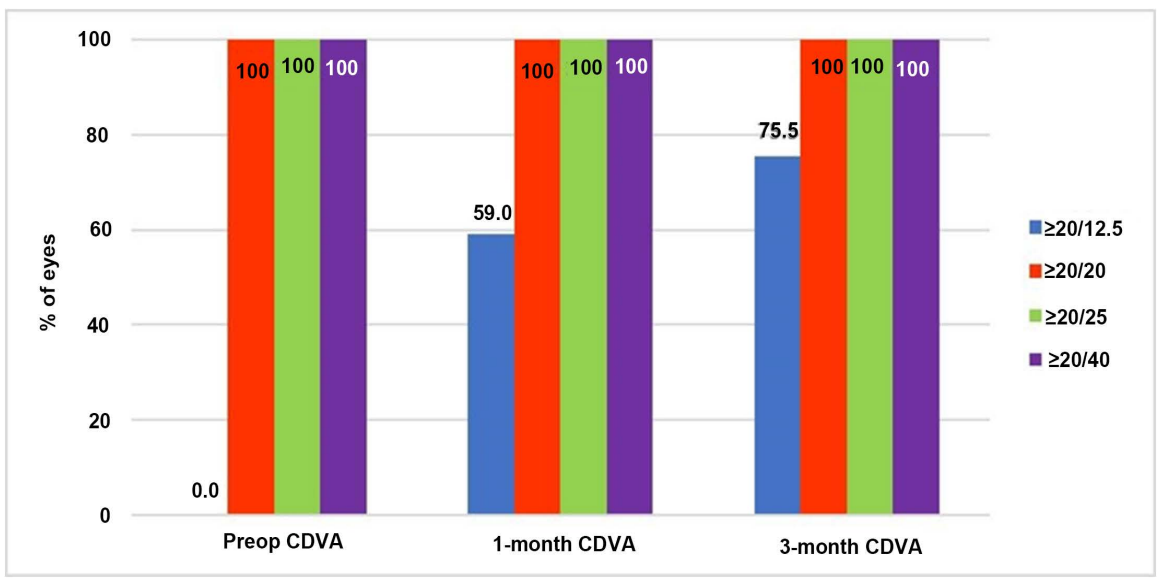

Figure 4. Preoperative and postoperative distribution of CDVA data.

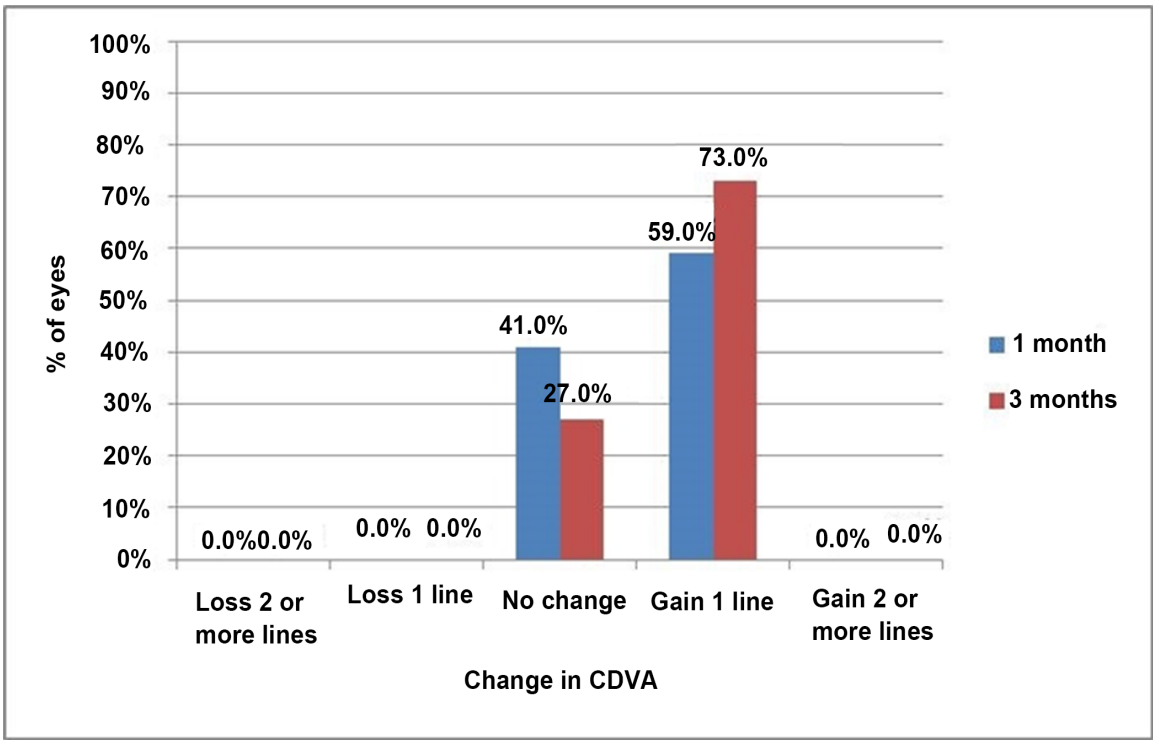

Figure 5. Distribution of the change with surgery in CDVA at 1 and 3 months postoperatively in the analyzed sample. 
Table 3. Preoperative and postoperative aberrometric data.

\begin{tabular}{cccc}
\hline $\begin{array}{c}\text { Mean }(\mathrm{SD}) \\
\text { Median }(\text { Range })\end{array}$ & Preoperative & 3-month postoperative & p-value \\
\hline Total RMS $(\mu \mathrm{m})$ & $4.43(1.42)$ & $0.70(0.59)$ & $<0.001$ \\
& $4.49(1.63$ to 8.22$)$ & $0.54(0.16$ to 4.20$)$ & \\
HOA RMS $(\mu \mathrm{m})$ & $0.20(0.06)$ & $0.23(0.10)$ & 0.050 \\
& $0.19(0.09$ to 0.44$)$ & $0.21(0.06$ to 0.67$)$ & \\
Primary coma RMS $(\mu \mathrm{m})$ & $0.10(0.06)$ & $0.15(0.11)$ & $<0.001$ \\
& $0.09(0.01$ to 0.28$)$ & $0.12(0.01$ to 0.64$)$ & \\
Spherical aberration $(\mu \mathrm{m})$ & $-0.05(0.11)$ & $0.03(0.08)$ & $<0.001$ \\
\hline
\end{tabular}

Abbreviations: RMS, root mean square; HOA, high order aberrations; SD, standard deviation; $\mu \mathrm{m}$, microns.

coma-like aberrations,resulting in a deterioration of visual qualityleading to patient dissatisfaction [3] [10] [11]. The development of wavefront technology in order to characterize ocular aberrations has increased the interest on how refractive surgery procedures can modify and improve the eye's optical quality. The main aim of this technology is not only to eliminate low-order aberrations (LOAs) but to minimize the induction of HOAs [12]. This study has been designed to report the visual, refractive, and aberrometric outcomes following wavefront-guided myopic LASIK using a high-resolution Hartmann-Shack aberrometer.

Conventional LASIK seems to induce more significant HOAs than PRK due to the flap creation [13]. Furthermore, wavefront-guided PRK is associated to fewer induced HOAs than wavefront-guided LASIK [13]. Yang et al. [14] attributes better visual performance in terms of HOAs after Epi-LASIK than after LASIK for large pupil diameters to differences in wound-healing processes between the two methods. On the other hand, it has been proposed that wavefront-guided treatments are not the best option for all patients undergoing photo ablative refractive surgery, [15] and only patients with high preoperative levels of HOAs $(>0.3 \mu \mathrm{m})$ would benefit [14]. Factors negatively affecting the success of LASIK treatments are rotational misalignments, which lead to a reduction in the effectiveness of astigmatic correction, and directional misalignments that induce coma-like aberration and secondary astigmatism [16] [17] [18]. The aberrometer used in the current series is based on advanced Fourier algorithms to characterize the aberrometric profile of the eye and offers iris registration that compensates for the pupil centroid shift and torsional movements for alignment in a supine position.

In our series, we have obtained excellent visual and refractive outcomes, confirming the efficacy and safety of the procedure performed. Various authors have reported excellent visual and refractive results also with the VISX excimer laser platform using wavefront-guided myopic treatments [3] [18] [19] [20] [21] [22] and some of them have studied the change in HOAs using different aberrometers. Jabbur et al. [20] found no induction of total ocular HOAs, SA and coma-like aberration after 6 months in a sample of 85 myopic eyes undergoing 
wavefront-guided LASIK using the Waves can aberrometric system, as in our series. Awwad et al. [23] reported a significant increase of total HOAs, SA, and coma-like aberration after wavefront LASIK with the VISX CustomVue system but none of the changes was statistically significant 3 months after surgery in a sample of 50 myopic eyes. However, Moshirfar and colleagues [3] found a statistically significant increase of total ocular HOAs, SA and coma-like aberration after myopic wavefront guided LASIK (increase factor 1.74) and PRK (increase factor 1.22) in a sample of 53 and 54 eyes, respectively, using the VISX Star S4 CustomVue platform at a 6-month endpoint. Significant differences between both groups were attributed to the flap as a possible source of HOAs [3]. Tanzer et al. [24] found no change in the induction of coma-like aberration and a statistically, but clinically not significant increase of total HOAs and SA in a sample of 497 myopic eyes operated with the VISX Star S4 IR excimer laser after a 3-month follow-up.

\section{Conclusion}

In summary, our results agree with the majority of the mentioned studies. The combination of the VISX S4 laser platform and the iDesign aberrometry with iris registration provides excellent visual and refractive outcomes 3 months after surgery with high levels of safety, efficacy and predictability. The outcomes from this prospective study indicate that wavefront-guided myopic LASIK with an ablation profile calculated according to the iDesign aberrometer yields no clinically significant induction of HOAs and, thus, is an efficacious and safe option for the preservation of visual acuity.Future studies evaluating the same parameters on a different cohort of patients (hyperopia and mixed astigmatism) would be desirable to corroborate these results.

\section{Statement}

The study reported in this publication was supported by a grant from Johnson \& Johnson Vision.

\section{Financial Disclosure}

The authors have no proprietary interest in any of the materials mentioned in this article.

\section{References}

[1] Hersh, P.S., Fry, K. and Blaker, J.W. (2003) Spherical Aberration after Laser In Situ Keratomileusis and Photorefractive Keratectomy. Clinical Results and Theoretical Models of Etiology. Journal of Cataract \& Refractive Surgery, 29, 2096-2104. https://doi.org/10.1016/j.jcrs.2003.09.008

[2] Bailey, M.D., Mitchell, G.L., Dhaliwal, D.K., Boxer Wachler, B.S. and Zadnik, K. (2003) Patient Satisfaction and Visual Symptoms after Laser In Situ Keratomileusis. Ophthalmology, 110, 1371-1378. https://doi.org/10.1016/S0161-6420(03)00455-X

[3] Moshirfar, M., Schliesser, J.A., Chang, J.C., Oberg, T.J., Mifflin, M.D., Townley, R., 
Livingston, M.K. and Kurz, C.J. (2010) Visual Outcomes after Wavefront-Guided Photorefractive Keratectomy and Wavefront-Guided Laser in Situ Keratomileusis: Prospective Comparison. Journal of Cataract \& Refractive Surgery, 36, 1336-1343. https://doi.org/10.1016/j.jcrs.2010.02.012

[4] Feng, Y., Yu, J. and Wang, Q. (2011) Meta-Analysis of Wavefront-Guided vs. Wavefront-Optimized LASIK for Myopia. Optometry and Vision Science, 88, 1463-1469.

[5] Schallhorn, S., Brown, M., Venter, J., Teenan, D., Hettinger, K. and Yamamoto, H. (2014) Early Clinical Outcomes of Wavefront-Guided Myopic LASIK Treatments Using a New-Generation Hartmann-Shack Aberrometer. Journal of Refractive Surgery, 30, 14-21.

[6] Stevens, J., Giubilei, M., Ficker, L. and Rosen, P. (2002) Prospective Study of Photorefractive Keratectomy for Myopia Using the Visx Star S2 Excimer Laser System. Journal of Refractive Surgery, 18, 502-508.

[7] Mifflin, M.D., Hatch, B.B., Sikder, S., Bell, J., Kurz, C.J. and Moshirfar, M. (2012) Custom versus Conventional PRK: A Prospective, Randomized, Contralateral Eye Comparison of Postoperative Visual Function. Journal of Refractive Surgery, 28, 127-132. https://doi.org/10.3928/1081597X-20120103-01

[8] Binder, P.S. and Rosenshein, J. (2007) Retrospective Comparison of 3 Laser Platforms to Correct Myopic Spheres and Spherocylinders Using Conventional and Wavefront Guided Treatments. Journal of Cataract \& Refractive Surgery, 33, 1158-1117. https://doi.org/10.1016/j.jcrs.2007.03.040

[9] Moshirfar, M., Shah, T.J., Skanchy, D.F., Linn, S.H. and Durrie, D.S. Meta-Analysis of the FDA Reports on Patient Reported Outcomes Using the Three Latest Platforms for LASIK (2017), Journal of Cataract \& Refractive Surgery, 33, 362-368. https://doi.org/10.3928/1081597X-20161221-02

[10] Holladay, J.T., Dudeja, D.R. and Chang, J. (1999) Functional Vision and Corneal Changes after Laser In Situ Keratomileusis Determined by Contrast Sensitivity, Glare Testing and Corneal Topography. Journal of Cataract \& Refractive Surgery, 25, 663-669. https://doi.org/10.1016/S0886-3350(99)00011-5

[11] Chalita, M.R., Chavala, S., Xu, M. and Krueger, R.R. (2004) Wavefront Analysis in Post-LASIK Eyes and Its Correlation with Visual Symptoms, Refraction and Topography. Ophthalmology, 111, 447-453. https://doi.org/10.1016/j.ophtha.2003.06.022

[12] Mrochen, M., Donetzky, C., Wüllner, C. and Löffler, J. (2004) Wavefront-Optimized Ablation Profiles: Theoretical Background. Journal of Cataract \& Refractive Surgery, 30, 775-785. https://doi.org/10.1016/j.jcrs.2004.01.026

[13] Waheed, S., Chalita, M.R., Xu, M. and Krueger, R.R. (2005) Flap-Induced and Laser-Induced Ocular Aberrations in a Two-Step LASIK Procedure. Journal of Refractive Surgery, 21, 346-352.

[14] Yang, X., Wang, Y., Zhao, K. and Fang, L. (2010) Comparison of Higher-Order Aberration and Optical Quality after Epi-LASIK and LASIK for Myopia. Graefe's Archive for Clinical and Experimental Ophthalmology, 249, 281-288. https://doi.org/10.1007/s00417-010-1394-x

[15] Artal, P. (2005) What Aberration Pattern (if any) Produces the Best Vision? 6th International Congress on Wavefront Sensing and Optimized Refractive Corrections, Athens, 11-13 February 2005.

[16] Swami, A.U., Steinert, R.F., Osborne, W.E. and White, A.A. (2002) Rotational Malposition during Laser in Situ Keratomileusis. American Journal of Ophthalmology, 133, 561-562. https://doi.org/10.1016/S0002-9394(01)01401-5 
[17] Chernyak, D.A. (2005) Iris-Based Cyclotorsional Image Alignment Method for Wavefront Registration. IEEE Transactions on Biomedical Engineering, 52, 2032-2040. https://doi.org/10.1109/TBME.2005.857674

[18] Kim, H. and Joo, C.K. (2008) Ocular Cyclotorsion According to Body Position and Flap Creation before Laser in Situ Keratomileusis. Journal of Cataract \& Refractive Surgery, 34, 557-561. https://doi.org/10.1016/j.jcrs.2007.11.030

[19] Khalifa, M., El-Kateb, M. and Shaheen, M.S. (2009) Iris Registration in Wavefront-Guided LASIK to Correct Mixed Astigmatism. Journal of Cataract \& Refractive Surgery, 35, 433-437. https://doi.org/10.1016/j.jcrs.2008.11.039

[20] Jabbur, N.S. and Kraff, C. (2005) Wavefront-Guided Laser in Situ Keratomileusis Using the WaveScan System for the Correction of Low to Moderate Myopia with Astigmatism: 6-Month Results in 277 Eyes. Journal of Cataract \& Refractive Surgery, 31, 1493-1501. https://doi.org/10.1016/j.jcrs.2004.12.050

[21] Schallhorn, S.C. and Venter, J.A. (2009) One-Month Outcomes of Wavefront-Guided LASIK for Low to Moderate Myopia with the VISX STAR S4 Laser in 32,569 Eyes. Journal of Refractive Surgery, 25, S634-S641.

[22] Pérez-Straziota, C.E., Randleman, B. and Stulting, R.D. (2010) Visual Acuity and Higher-Order Aberrations with Wavefront-Guided and Wavefront-Optimized Laser in Situ Keratomileusis. Journal of Cataract \& Refractive Surgery, 36, 437-441. https://doi.org/10.1016/j.jcrs.2009.09.031

[23] Awwad, S.T., Bowman, R.W., Cavanagh, H.D. and McCulley, J.P. (2007) Wavefront-Guided LASIK for Myopia Using the LADAR CustomCornea and the VISX CustomVue. Journal of Refractive Surgery, 23, 26-38.

[24] Tanzer, D.J., Brunstetter, T., Zeber, R., Hofmeister, T., Kaupp, S., Kelly, N., Mirzaoff, M., Sray, W., Brown, M. and Schallhorn, S. (2013) Laser in Situ Keratomileusis in United States Navalaviators. Journal of Cataract \& Refractive Surgery, 39, 1047-1058. https://doi.org/10.1016/j.jcrs.2013.01.046 